\title{
Development of Martian Regolith Simulants \\ In Situ Resource Availability And Potential
}

\author{
Laura E. Fackrell
}




\section{Mars Simulants and Exploration}

Appropriate analog materials are an essential tool in the studies of the Martian surface and mission preparation

Growth Experiment

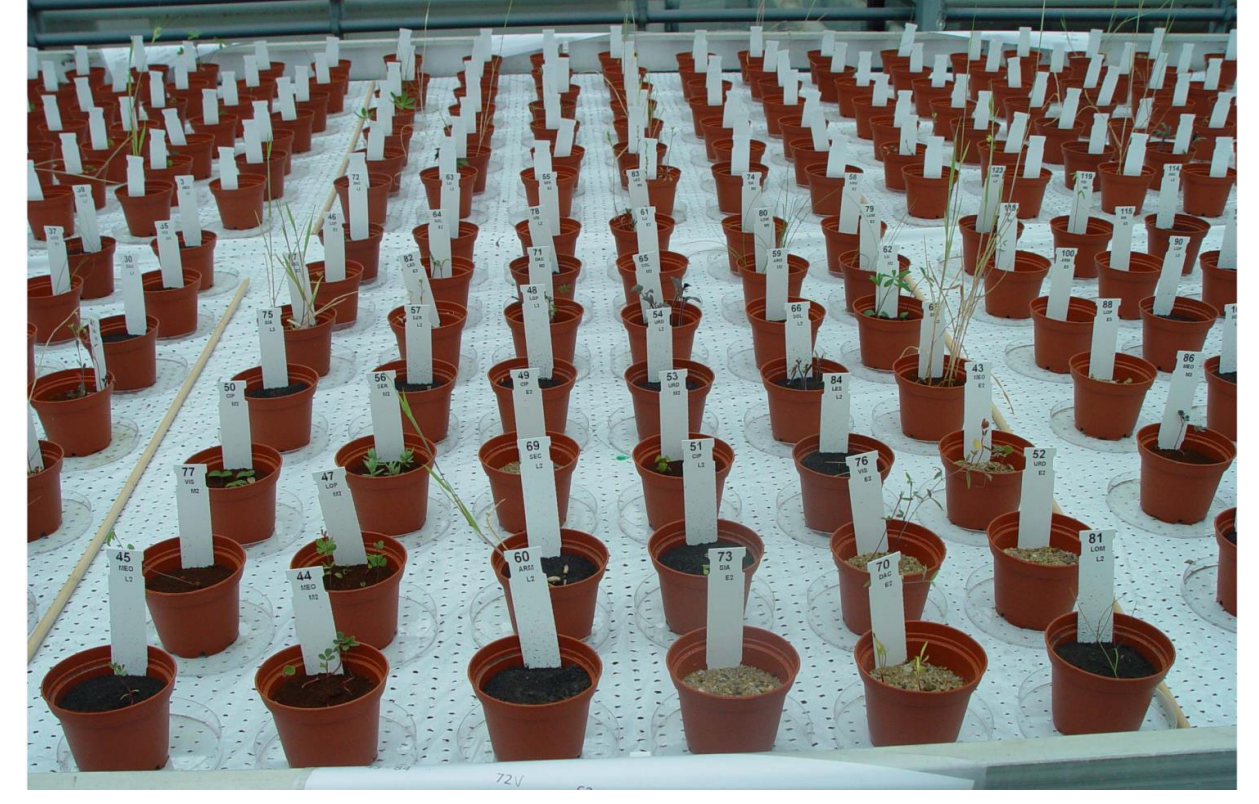

Wamelink et al. 2014

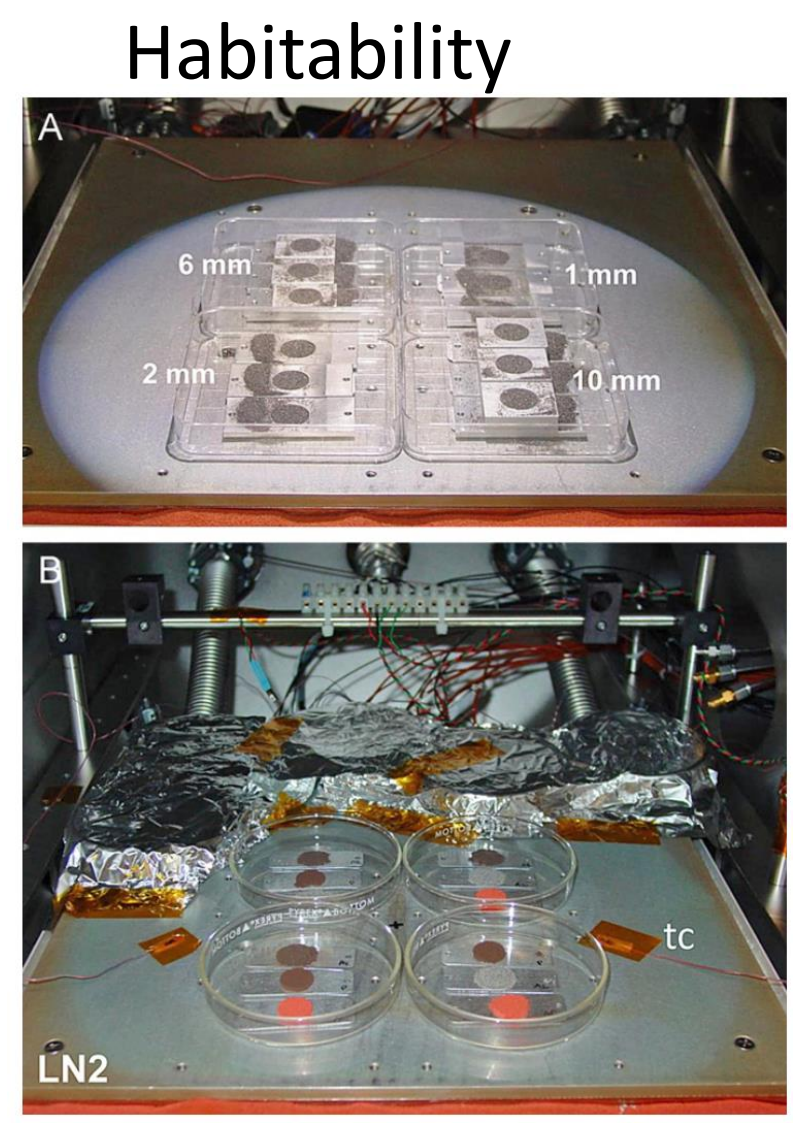

Schuerger et al. 2012
Rover Testing

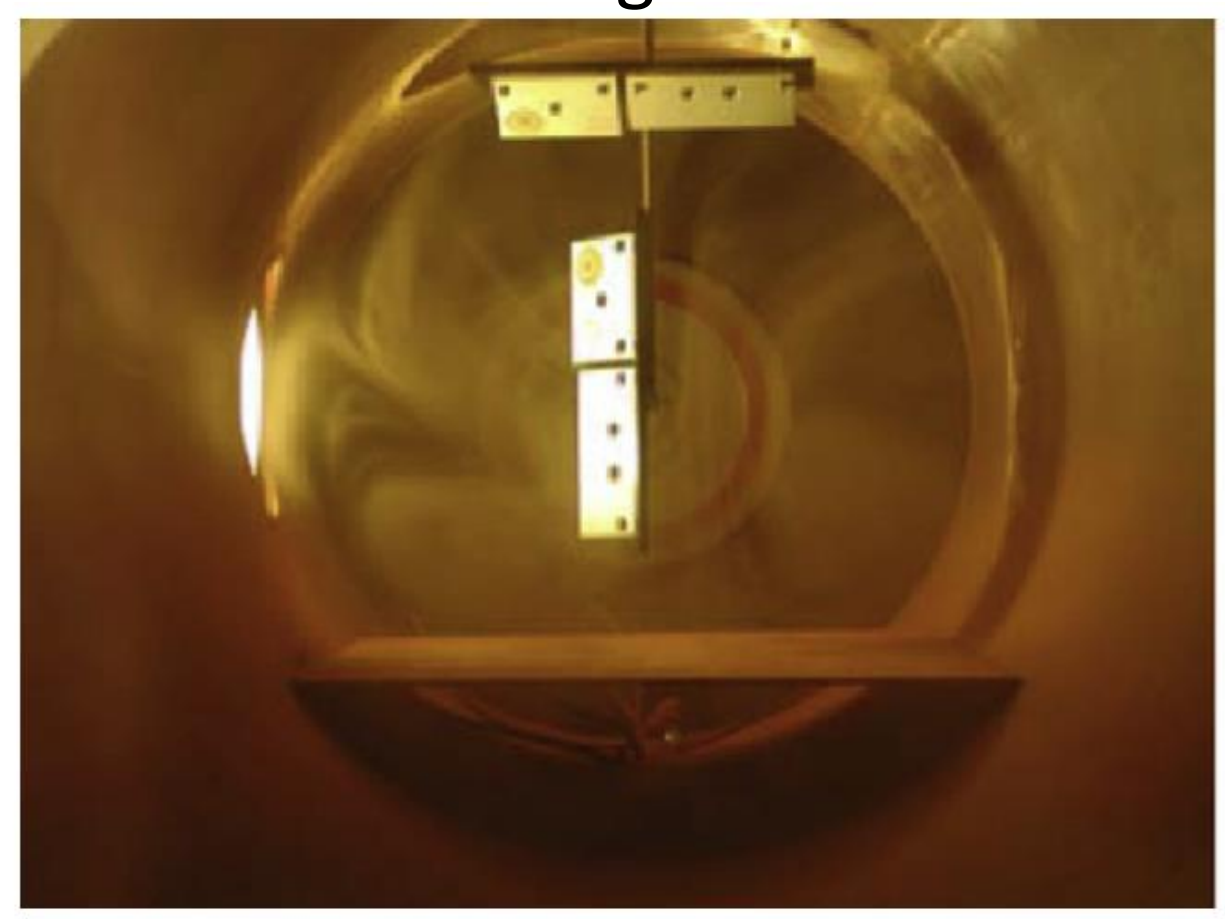

Seiferlin et al. 2008 


\section{Martian Simulant Development}

Application

Characteristics

Volume

Materials

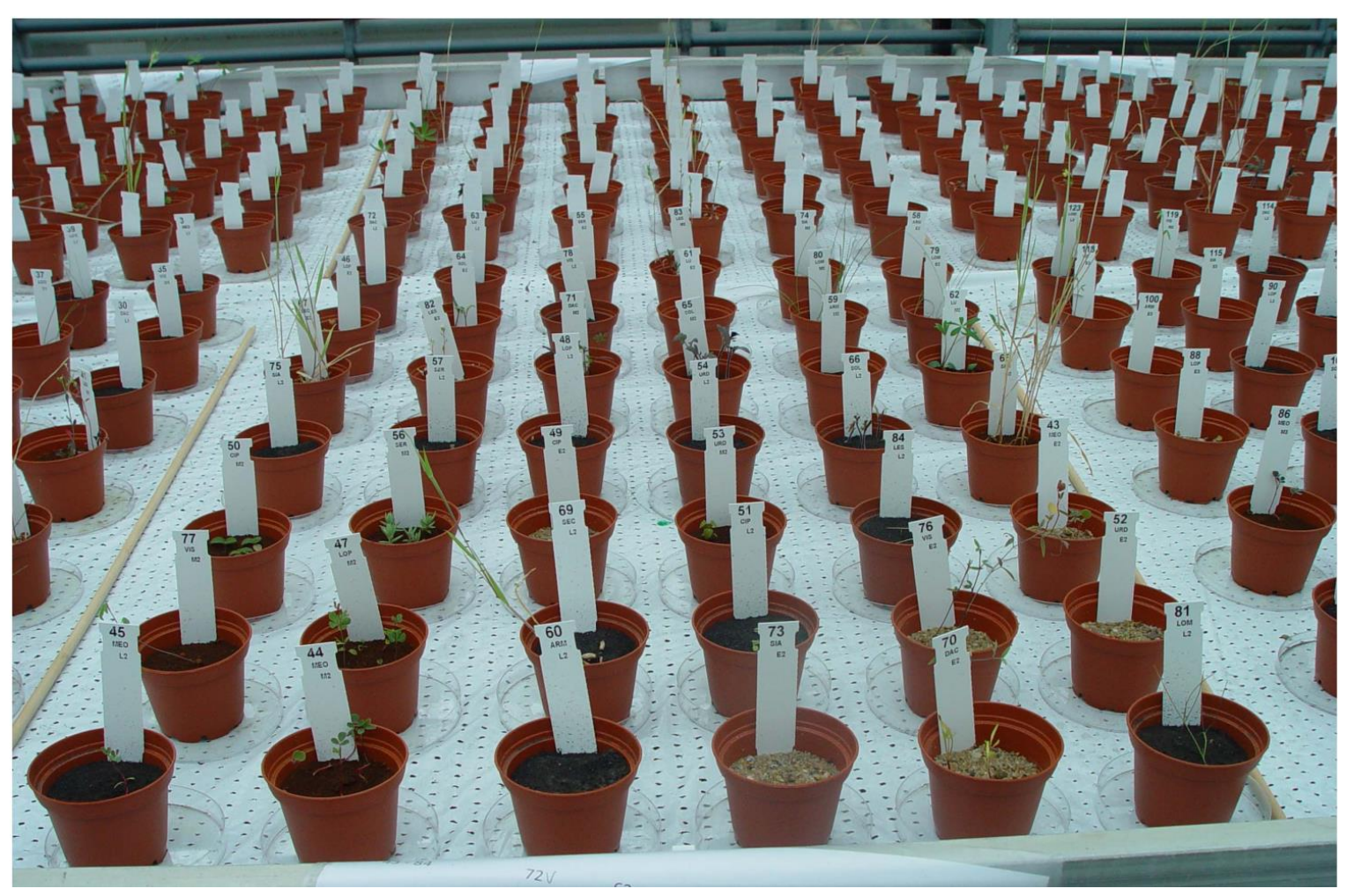

Wamelink et al. 2014 


\section{Martian Simulant Development}

Application

- Plant Growth Experiment

Characteristics

- Chemical

- Mineral

- Growth Limiting Conditions (Salinity, toxicitiy)

Volume

- Several Kg

Materials

- Field Samples

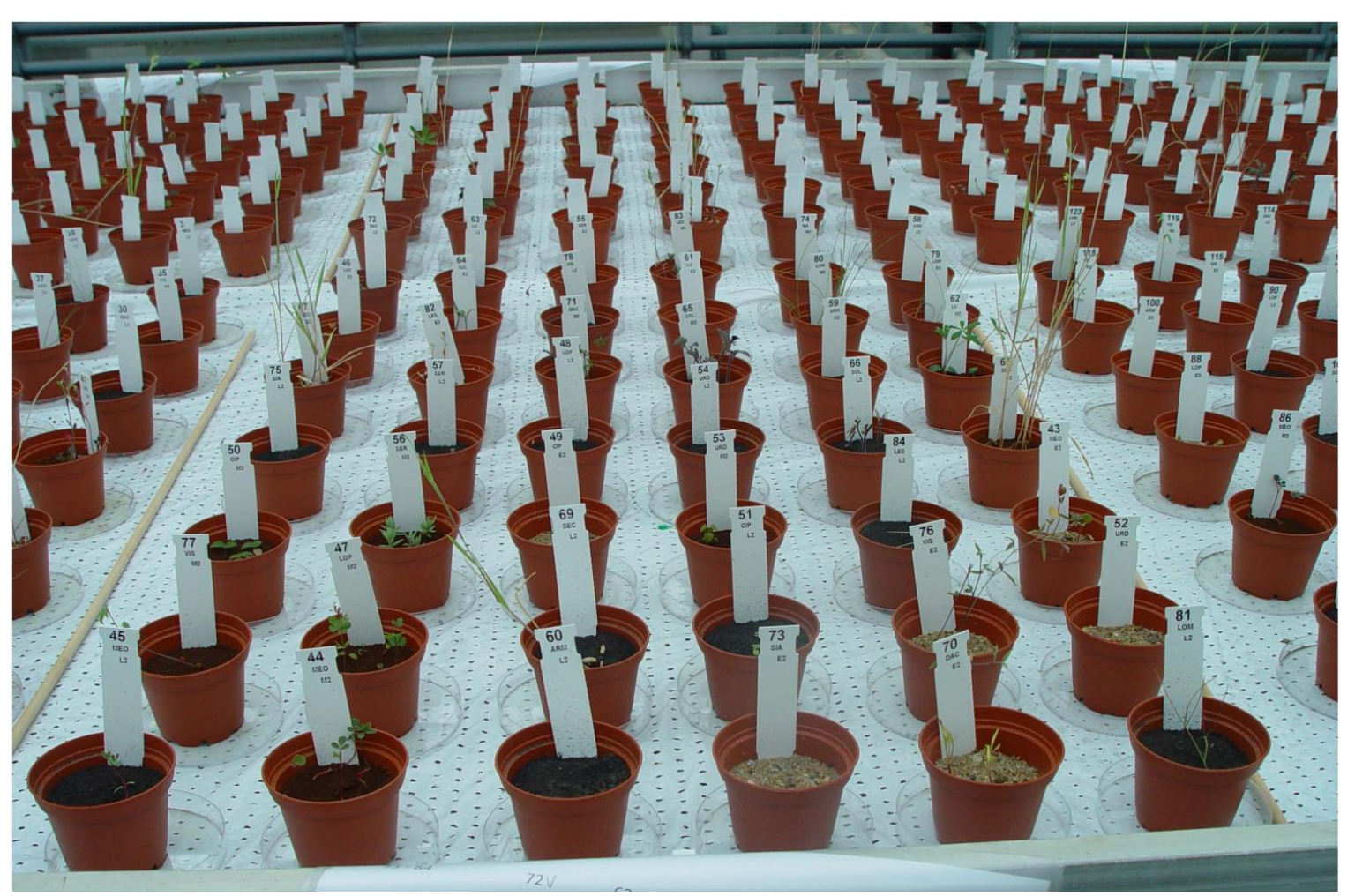

Wamelink et al. 2014

- Purchased in Bulk 


\section{Methods: Composition Determination}

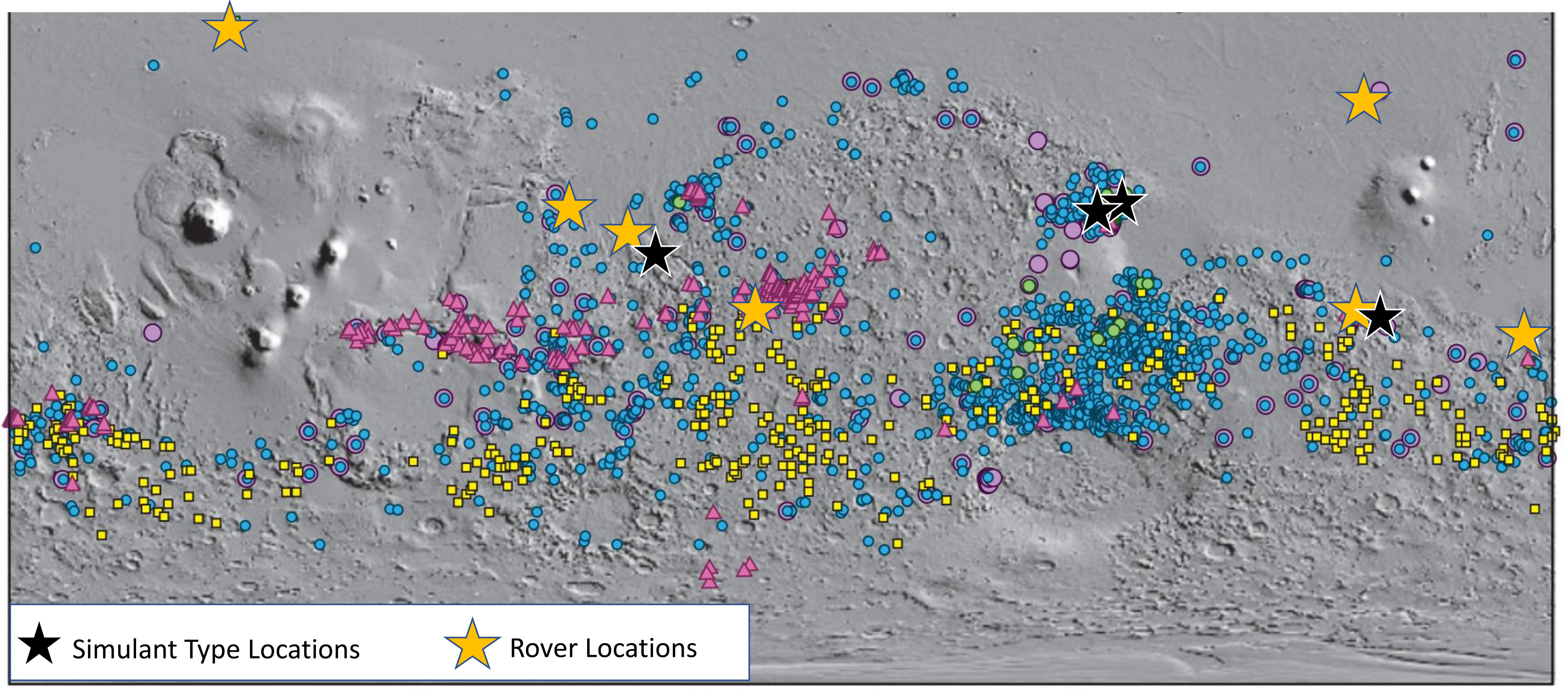


Table 1: Martian Regolith Components

\begin{tabular}{|c|c|c|c|c|c|}
\hline Components & $\begin{array}{l}\text { Regolith 1: Basalt } \\
\text { (unaltered) }\end{array}$ & Regolith 2: Sulfate & $\begin{array}{l}\text { Regolith 3: } \\
\text { Phyllosilicate I }\end{array}$ & $\begin{array}{l}\text { Regolith 4: } \\
\text { Phyllosilicate II }\end{array}$ & Regolith 5: Carbonate \\
\hline $\begin{array}{l}\text { Basalts and Primary } \\
\text { Silicates }\end{array}$ & $\begin{array}{l}\text { Fresh basalt }^{1} \\
\text { Olivine }^{3}\end{array}$ & Non-Fresh basalt ${ }^{2}$ & Non-Fresh Basalt ${ }^{2}$ & Non-Fresh Basalt ${ }^{2}$ & $\begin{array}{l}\text { Non-Fresh Basalt }{ }^{2} \\
\text { Olivine }^{5}\end{array}$ \\
\hline
\end{tabular}
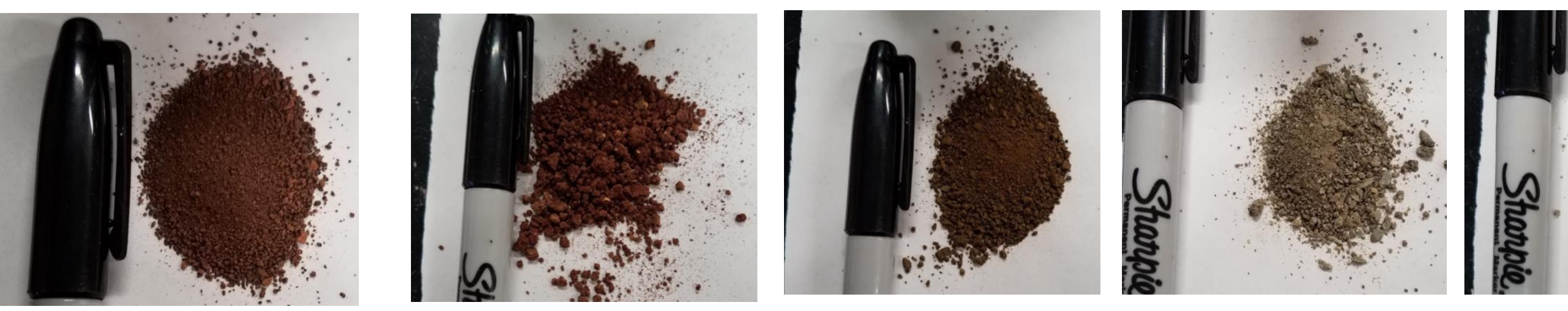
Table 1: Martian Regolith Components

\begin{tabular}{|c|c|c|c|c|c|}
\hline Components & $\begin{array}{l}\text { Regolith 1: Basalt } \\
\text { (unaltered) }\end{array}$ & Regolith 2: Sulfate & $\begin{array}{l}\text { Regolith 3: } \\
\text { Phyllosilicate I }\end{array}$ & $\begin{array}{l}\text { Regolith 4: } \\
\text { Phyllosilicate II }\end{array}$ & Regolith 5: Carbonate \\
\hline $\begin{array}{l}\text { Basalts and Primary } \\
\text { Silicates }\end{array}$ & $\begin{array}{l}\text { Fresh basalt }^{1} \\
\text { Olivine }^{3}\end{array}$ & Non-Fresh basalt ${ }^{2}$ & Non-Fresh Basalt ${ }^{2}$ & Non-Fresh Basalt ${ }^{2}$ & $\begin{array}{l}\text { Non-Fresh Basalt }{ }^{2} \\
\text { Olivine }^{5}\end{array}$ \\
\hline Phyllosilicates & - & - & Nontronite $^{3}$ & Chlorite $^{3}$ & Nontronite $^{3}$ \\
\hline
\end{tabular}
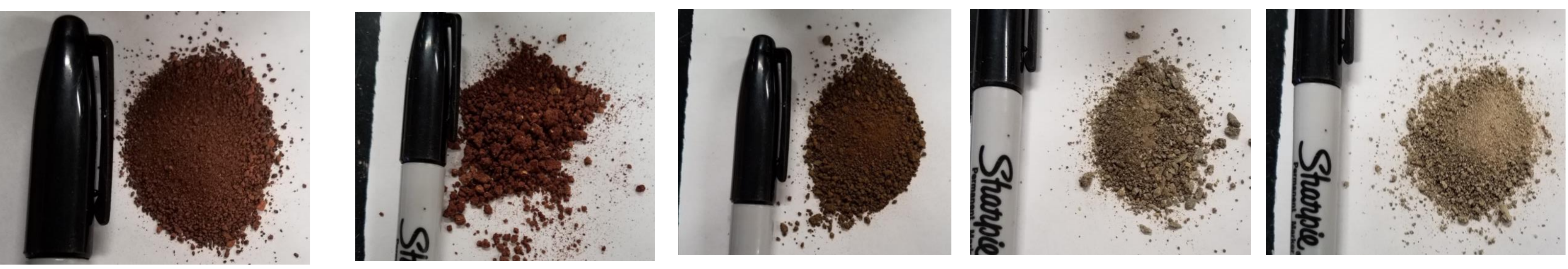
Table 1: Martian Regolith Components

\begin{tabular}{l|l|l|l|l|l}
\hline Components & $\begin{array}{l}\text { Regolith 1: Basalt } \\
\text { (unaltered) }\end{array}$ & Regolith 2: Sulfate & $\begin{array}{l}\text { Regolith 3: } \\
\text { Phyllosilicate I }\end{array}$ & $\begin{array}{l}\text { Regolith 4: } \\
\text { Phyllosilicate II }\end{array}$ \\
\hline $\begin{array}{l}\text { Basalts and Primary } \\
\text { Silicates }\end{array}$ & $\begin{array}{l}\text { Fresh basalt }^{1} \\
\text { Olivine }^{3}\end{array}$ & Non-Fresh basalt & & Non-Fresh Basalt & \\
\hline Phyllosilicates & - & - & Nontronite $^{3}$ & Non-Fresh Basalt $^{2}$ & $\begin{array}{l}\text { Non-Fresh Basalt }^{2} \\
\text { Olivine }^{5}\end{array}$ \\
\hline Sulfates & - & Copiapite $^{4}$ & - & Chlorite $^{3}$ & Nontronite \\
\hline
\end{tabular}
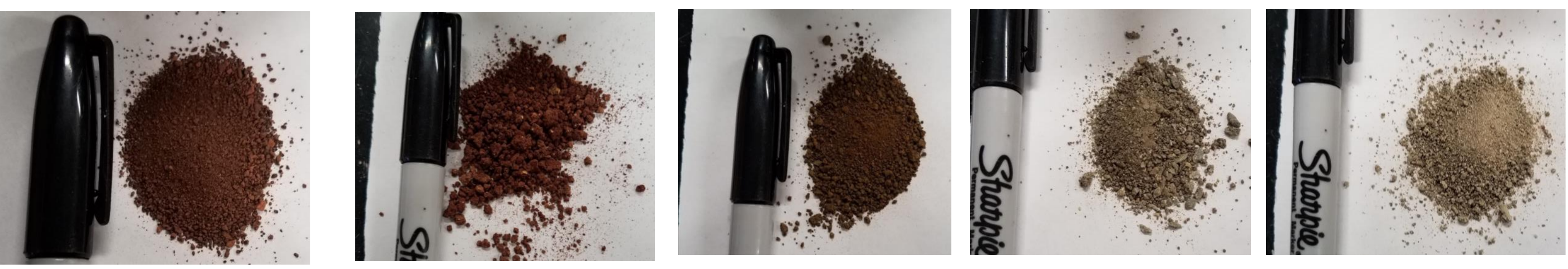
Table 1: Martian Regolith Components

\begin{tabular}{|c|c|c|c|c|c|}
\hline Components & $\begin{array}{l}\text { Regolith 1: Basalt } \\
\text { (unaltered) }\end{array}$ & Regolith 2: Sulfate & $\begin{array}{l}\text { Regolith 3: } \\
\text { Phyllosilicate I }\end{array}$ & $\begin{array}{l}\text { Regolith 4: } \\
\text { Phyllosilicate II }\end{array}$ & Regolith 5: Carbonate \\
\hline $\begin{array}{l}\text { Basalts and Primary } \\
\text { Silicates }\end{array}$ & $\begin{array}{l}\text { Fresh basalt }^{1} \\
\text { Olivine }^{3}\end{array}$ & Non-Fresh basalt ${ }^{2}$ & Non-Fresh Basalt ${ }^{2}$ & Non-Fresh Basalt ${ }^{2}$ & $\begin{array}{l}\text { Non-Fresh Basalt }{ }^{2} \\
\text { Olivine }^{5}\end{array}$ \\
\hline Phyllosilicates & - & - & Nontronite $^{3}$ & Chlorite $^{3}$ & Nontronite $^{3}$ \\
\hline Sulfates & - & Copiapite $^{4}$ & - & - & \\
\hline Carbonates & - & - & - & - & Magnesite $^{3}$ \\
\hline
\end{tabular}
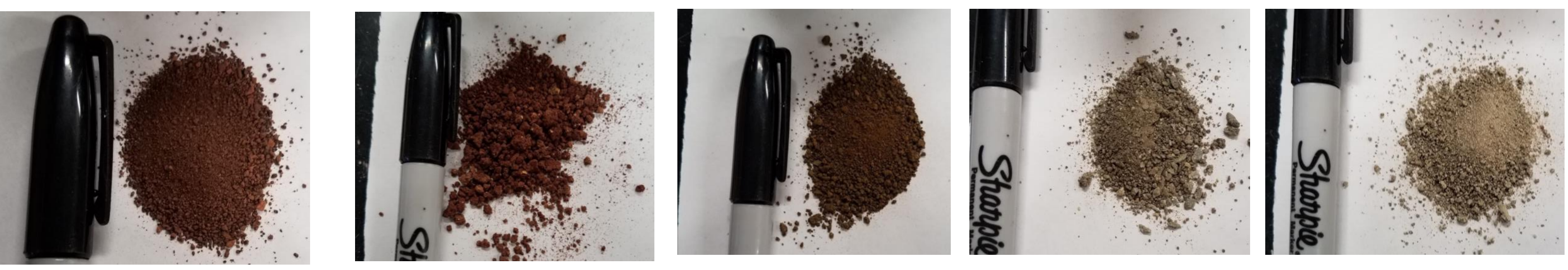
Table 1: Martian Regolith Components

\begin{tabular}{|c|c|c|c|c|c|}
\hline Components & $\begin{array}{l}\text { Regolith 1: Basalt } \\
\text { (unaltered) }\end{array}$ & Regolith 2: Sulfate & $\begin{array}{l}\text { Regolith 3: } \\
\text { Phyllosilicate I }\end{array}$ & $\begin{array}{l}\text { Regolith 4: } \\
\text { Phyllosilicate II }\end{array}$ & Regolith 5: Carbonate \\
\hline $\begin{array}{l}\text { Basalts and Primary } \\
\text { Silicates }\end{array}$ & $\begin{array}{l}\text { Fresh basalt }^{1} \\
\text { Olivine }^{3}\end{array}$ & Non-Fresh basalt ${ }^{2}$ & Non-Fresh Basalt ${ }^{2}$ & Non-Fresh Basalt ${ }^{2}$ & $\begin{array}{l}\text { Non-Fresh Basalt }{ }^{2} \\
\text { Olivine }^{5}\end{array}$ \\
\hline Phyllosilicates & - & - & Nontronite ${ }^{3}$ & Chlorite $^{3}$ & Nontronite $^{3}$ \\
\hline Sulfates & - & Copiapite $^{4}$ & - & - & \\
\hline Carbonates & - & - & - & - & Magnesite $^{3}$ \\
\hline Amorphous & $\begin{array}{l}\text { Volcanic Glass }{ }^{1} \text { and/or } \\
\text { Nano-phase ferric } \\
\text { oxides }^{5}\end{array}$ & $\begin{array}{l}\text { Hydrated silica }^{3} \\
\text { and Nano-phase ferric } \\
\text { oxides }^{5}\end{array}$ & Ferrihydrite/Goethite $^{5}$ & Ferrihydrite/Goethite ${ }^{5}$ & $\begin{array}{l}\text { Hydrous magnesium } \\
\text { silicates }^{5}\end{array}$ \\
\hline
\end{tabular}


Table 1: Martian Regolith Components

\begin{tabular}{|c|c|c|c|c|c|}
\hline Components & $\begin{array}{l}\text { Regolith 1: Basalt } \\
\text { (unaltered) }\end{array}$ & Regolith 2: Sulfate & $\begin{array}{l}\text { Regolith 3: } \\
\text { Phyllosilicate I }\end{array}$ & $\begin{array}{l}\text { Regolith 4: } \\
\text { Phyllosilicate II }\end{array}$ & Regolith 5: Carbonate \\
\hline $\begin{array}{l}\text { Basalts and Primary } \\
\text { Silicates }\end{array}$ & $\begin{array}{l}\text { Fresh basalt }^{1} \\
\text { Olivine }^{3} \\
\end{array}$ & Non-Fresh basalt ${ }^{2}$ & Non-Fresh Basalt ${ }^{2}$ & Non-Fresh Basalt ${ }^{2}$ & $\begin{array}{l}\text { Non-Fresh Basalt }{ }^{2} \\
\text { Olivine }^{5}\end{array}$ \\
\hline Phyllosilicates & - & - & Nontronite $^{3}$ & Chlorite $^{3}$ & Nontronite $^{3}$ \\
\hline Sulfates & - & Copiapite $^{4}$ & - & - & \\
\hline Carbonates & - & - & - & - & Magnesite $^{3}$ \\
\hline Amorphous & $\begin{array}{l}\text { Volcanic Glass }{ }^{1} \text { and/or } \\
\text { Nano-phase ferric } \\
\text { oxides }^{5}\end{array}$ & $\begin{array}{l}\text { Hydrated silica }{ }^{3} \\
\text { and Nano-phase ferric } \\
\text { oxides }^{5}\end{array}$ & Ferrihydrite/Goethite ${ }^{5}$ & Ferrihydrite/Goethite ${ }^{5}$ & $\begin{array}{l}\text { Hydrous magnesium } \\
\text { silicates }^{5}\end{array}$ \\
\hline Salts & $\begin{array}{l}\text { Anhydrite, Epsomite }{ }^{5} \\
\text { Perchlorate }{ }^{* 5}\end{array}$ & $\begin{array}{l}\text { Anhydrite, Epsomite }{ }^{5} \\
\text { Perchlorate*5 }\end{array}$ & $\begin{array}{l}\text { Anhydrite, Epsomite }{ }^{5} \\
\text { Perchlorate }\end{array}$ & $\begin{array}{l}\text { Anhydrite, Epsomite }{ }^{5} \\
\text { Perchlorate*5 }\end{array}$ & $\begin{array}{l}\text { Anhydrite, Epsomite } \\
\text { Perchlorate }\end{array}$ \\
\hline
\end{tabular}


Table 1: Martian Regolith Components

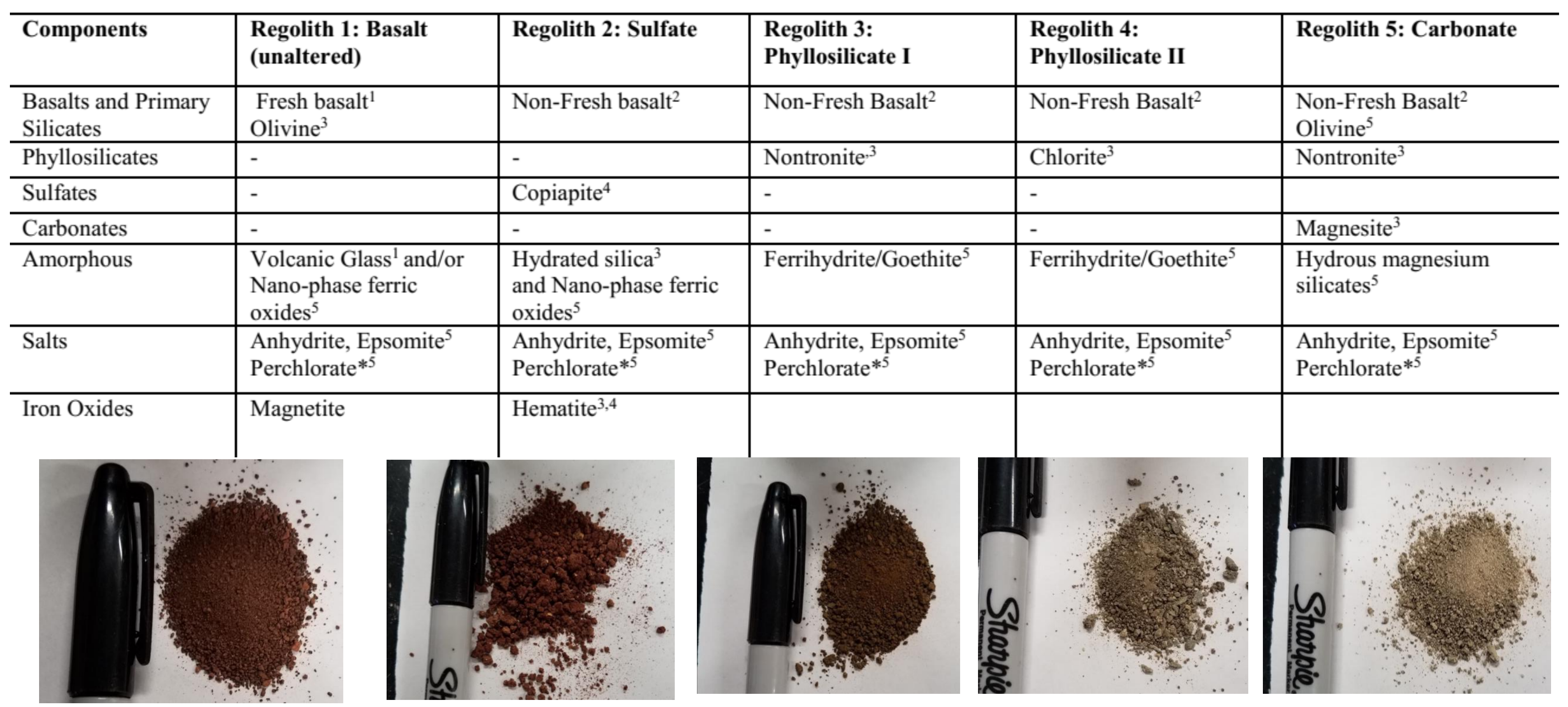


Table 1: Martian Regolith Components

\begin{tabular}{|c|c|c|c|c|}
\hline $\begin{array}{l}\text { Regolith 1: Basalt } \\
\text { (unaltered) }\end{array}$ & Regolith 2: Sulfate & $\begin{array}{l}\text { Regolith 3: } \\
\text { Phyllosilicate I }\end{array}$ & $\begin{array}{l}\text { Regolith 4: } \\
\text { Phyllosilicate II }\end{array}$ & Regolith 5: Carbonate \\
\hline $\begin{array}{l}\text { 90g NM Basalt } \\
2 \mathrm{~g}-\mathrm{FeO} \\
2 \mathrm{~g} \text { Anhydrite } \\
1 \mathrm{~g} \text { Epsomite/Perchlorate } \\
1 \mathrm{~g} \text { Magnetite } \\
\text { 4g Olivine } \\
\\
\text { Estimated Expected } \\
\text { Ratio } \\
65-70 \% \text { Primary } \\
\text { Minerals } \\
30 \% \text { Amorphous } \\
2 \% \text { Salts }\end{array}$ & $\begin{array}{l}\text { 45g EWash Basalt } \\
\text { 30g Copiapite** } \\
15 \mathrm{~g} \text { Hematite } \\
5 \mathrm{~g} \text { Nano-FeO } \\
2 \mathrm{~g} \text { Opal-CT } \\
2 \mathrm{~g} \text { Anhydrite } \\
1 \mathrm{~g} \text { Epsomite } \\
\text { Estimated Expected } \\
\text { Ratio } \\
40 \% \text { Primary Minerals } \\
20 \% \text { Sulfate } \\
10 \% \text { Iron Oxide } \\
30 \% \text { Amorphous } \\
2 \% \text { Salts }\end{array}$ & $\begin{array}{l}\text { 45g EWash Basalt } \\
\text { 32g Nontronite Swa-1 } \\
\text { 15g Ferrihydrate } \\
\text { 5g Magnetite } \\
\text { 2g Anhydrite } \\
\text { 1g Epsomite } \\
\\
\text { Estimated Expected } \\
\text { Ratio } \\
40 \% \text { Primary Minerals } \\
30 \% \text { Phyllosilicate } \\
30 \% \text { Amorphous } \\
2 \% \text { Salts }\end{array}$ & $\begin{array}{l}\text { 45g EWash Basalt } \\
\text { 32g Chlorite } \\
15 \mathrm{~g} \text { Illite } \\
5 \mathrm{~g} \text { Allophane } \\
2 \mathrm{~g} \text { Anhydrite } \\
1 \mathrm{~g} \text { Epsomite } \\
\text { 40\% Primary Minerals } \\
30 \% \text { Phyllosilicate } \\
30 \% \text { Amorphous } \\
2 \% \text { Salts }\end{array}$ & $\begin{array}{l}45 \mathrm{~g} \text { EWash Basalt } \\
25 \mathrm{~g} \text { Magnesite } \\
15 \mathrm{~g} \text { Notronite } \\
7 \mathrm{~g} \text { Olivine Dust } \\
5 \mathrm{~g} \text { Hydrous Mg Silicate } \\
2 \mathrm{~g} \text { Anhydrite } \\
1 \mathrm{~g} \text { Epsomite } \\
\text { 40\% Primary Minerals } \\
30 \% \text { Carbonate } \\
30 \% \text { Amorphous } \\
2 \% \text { Salts }\end{array}$ \\
\hline
\end{tabular}




\section{Methods: Analysis of Components}
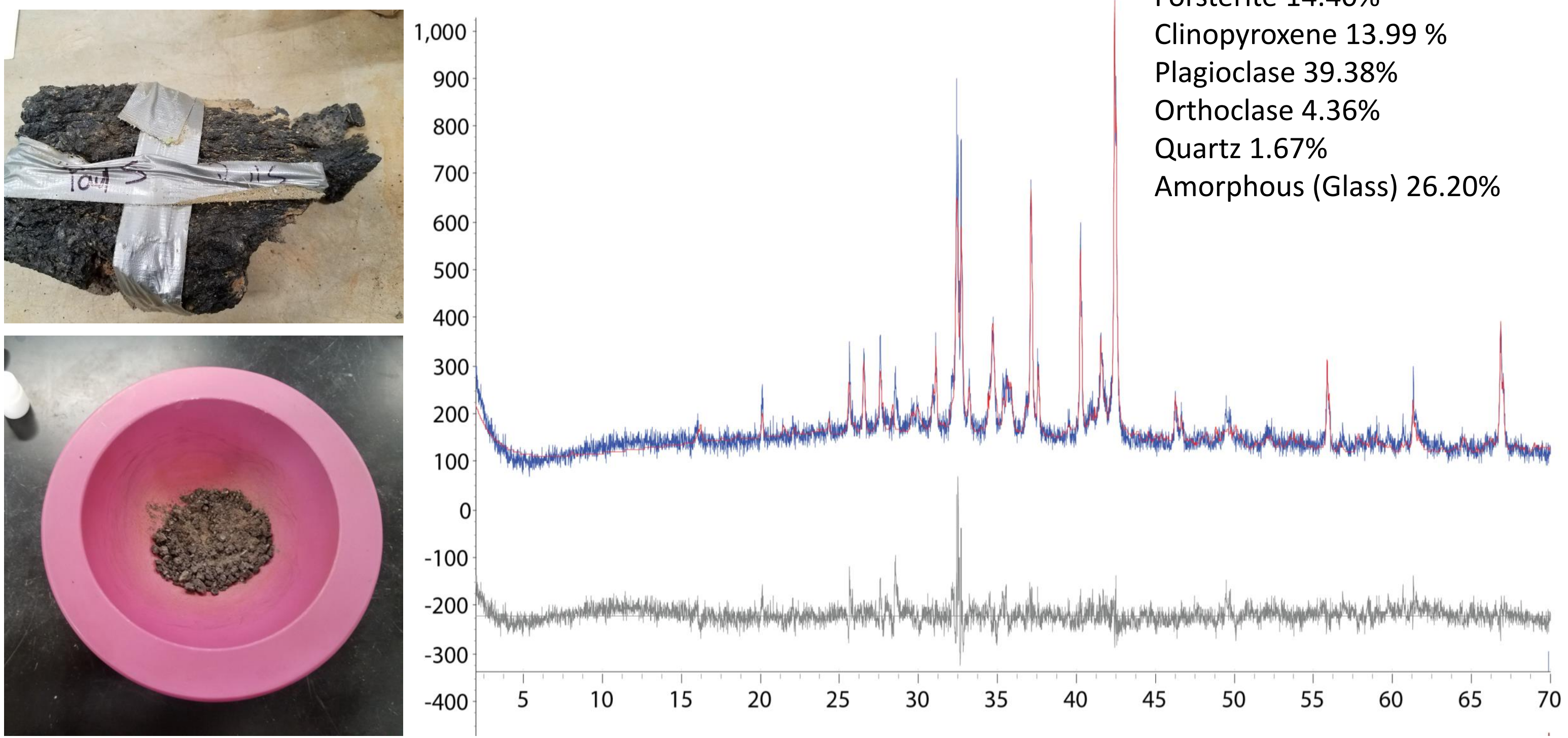


\section{Methods: Making Simulants}

- Components mechanically crushed and weighed

- All components except epsomite mixed together (Dry Ingredients)

- Epsomite was added in solution (Wet ingredients)

- This mud-like mixture dried in oven at $60^{\circ} \mathrm{C}$

- The dried 'mud' then crushed again
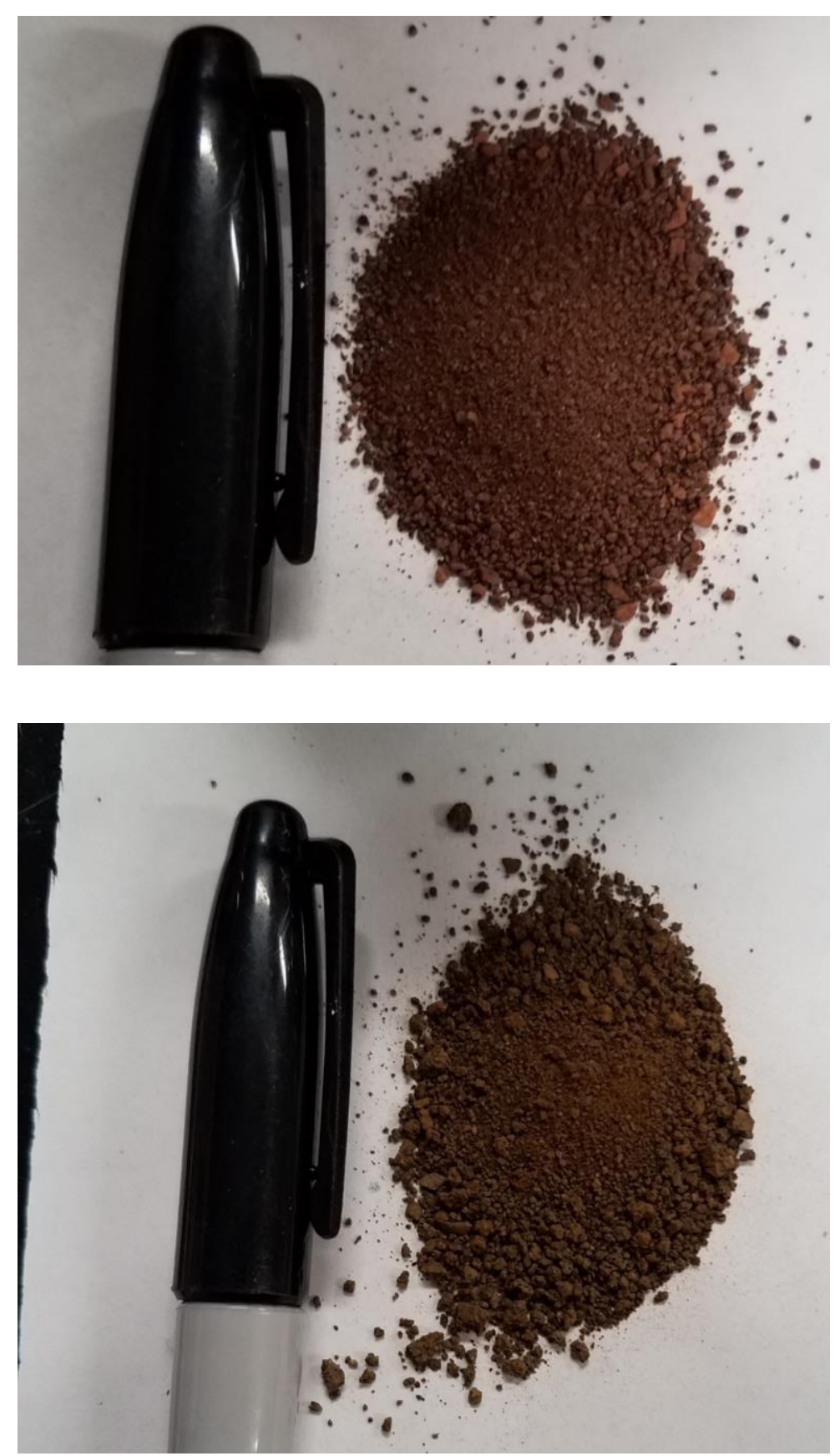


\section{Methods: Analysis of Simulants}

- Final Simulants analyzed with XRD and Reitveld analysis

- Additional XRF and Spectral analysis forthcoming

- Full pattern fitting is also intended to be applied for better constraint of disordered and amorphous phases
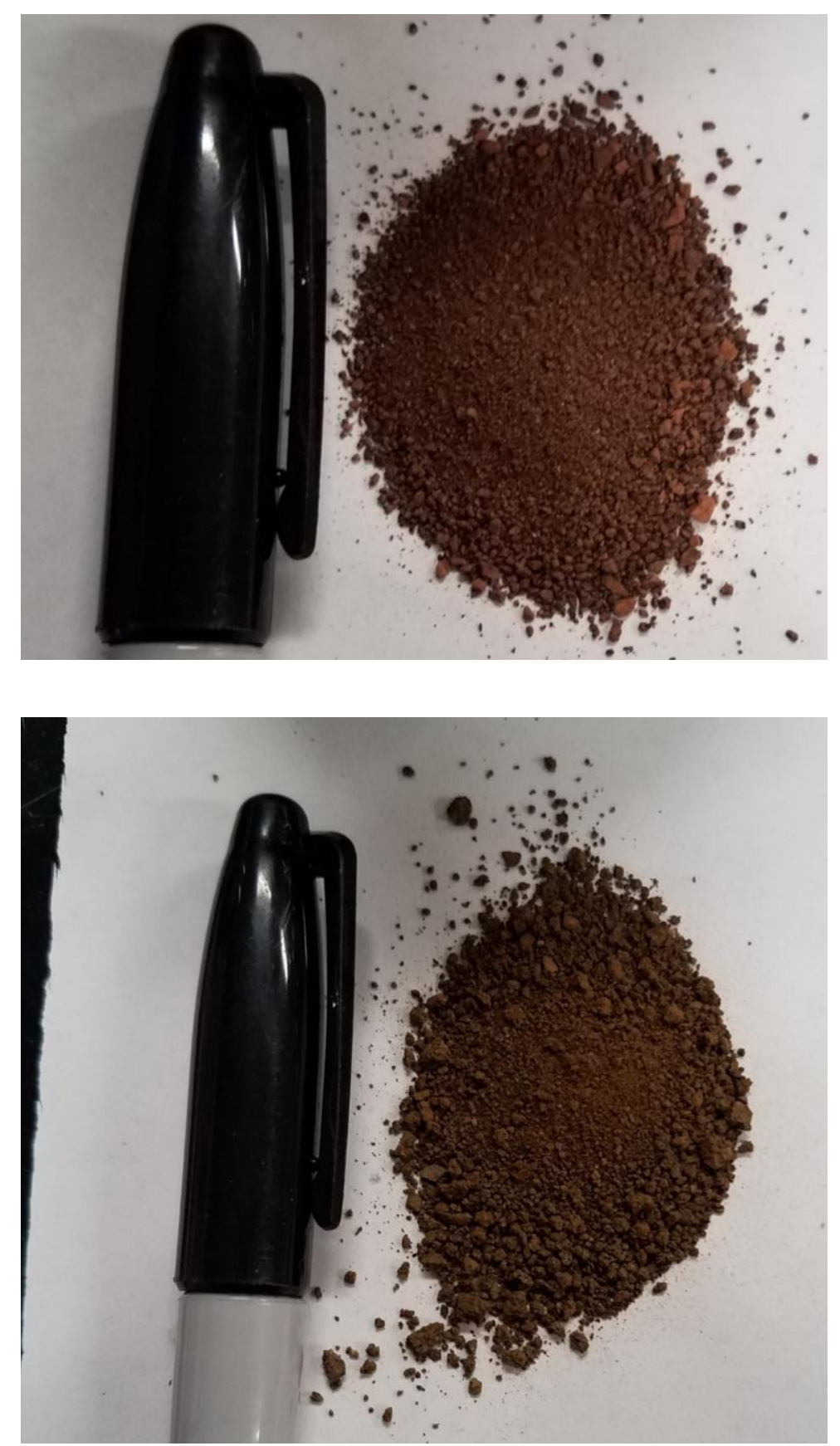


\section{Results}
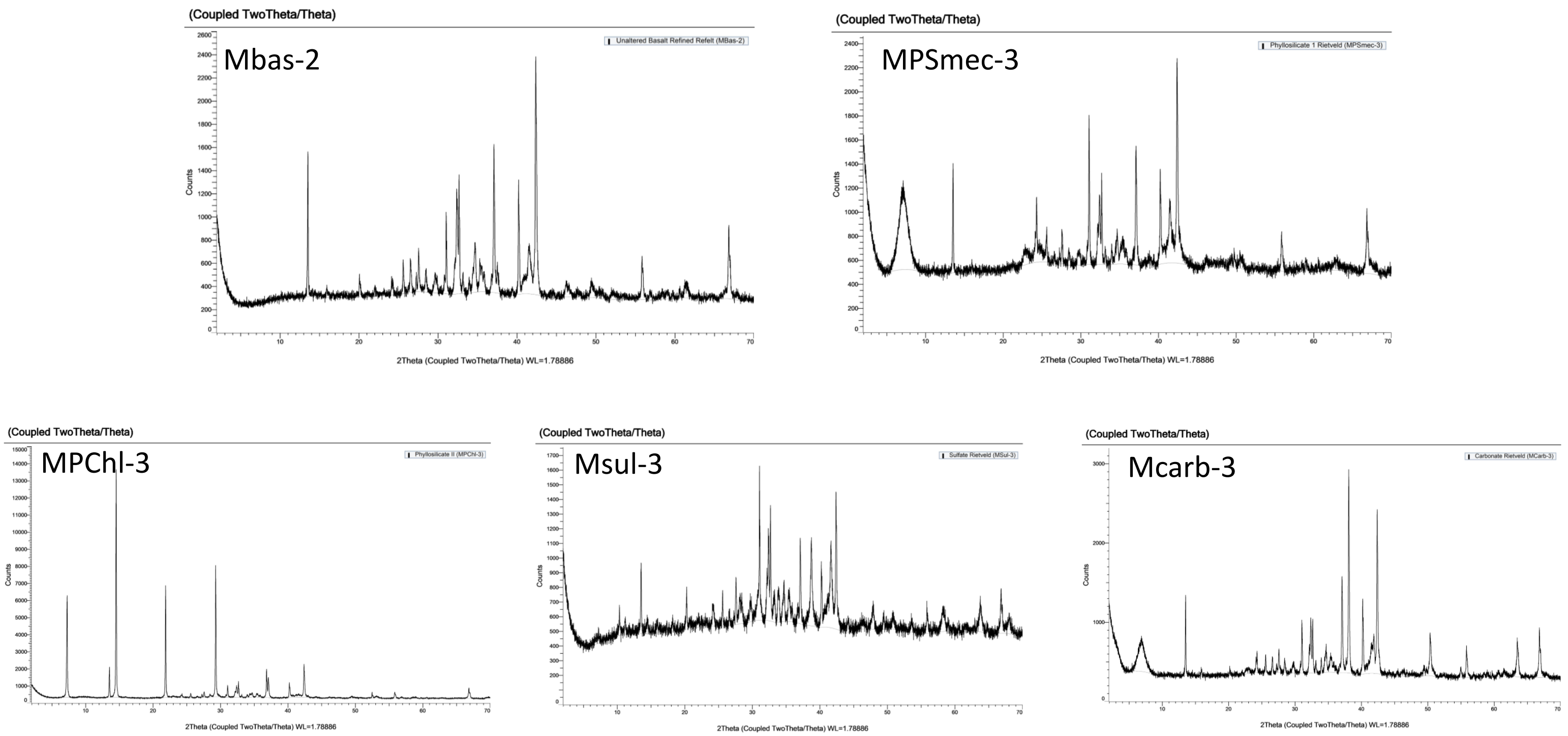


\section{Results}
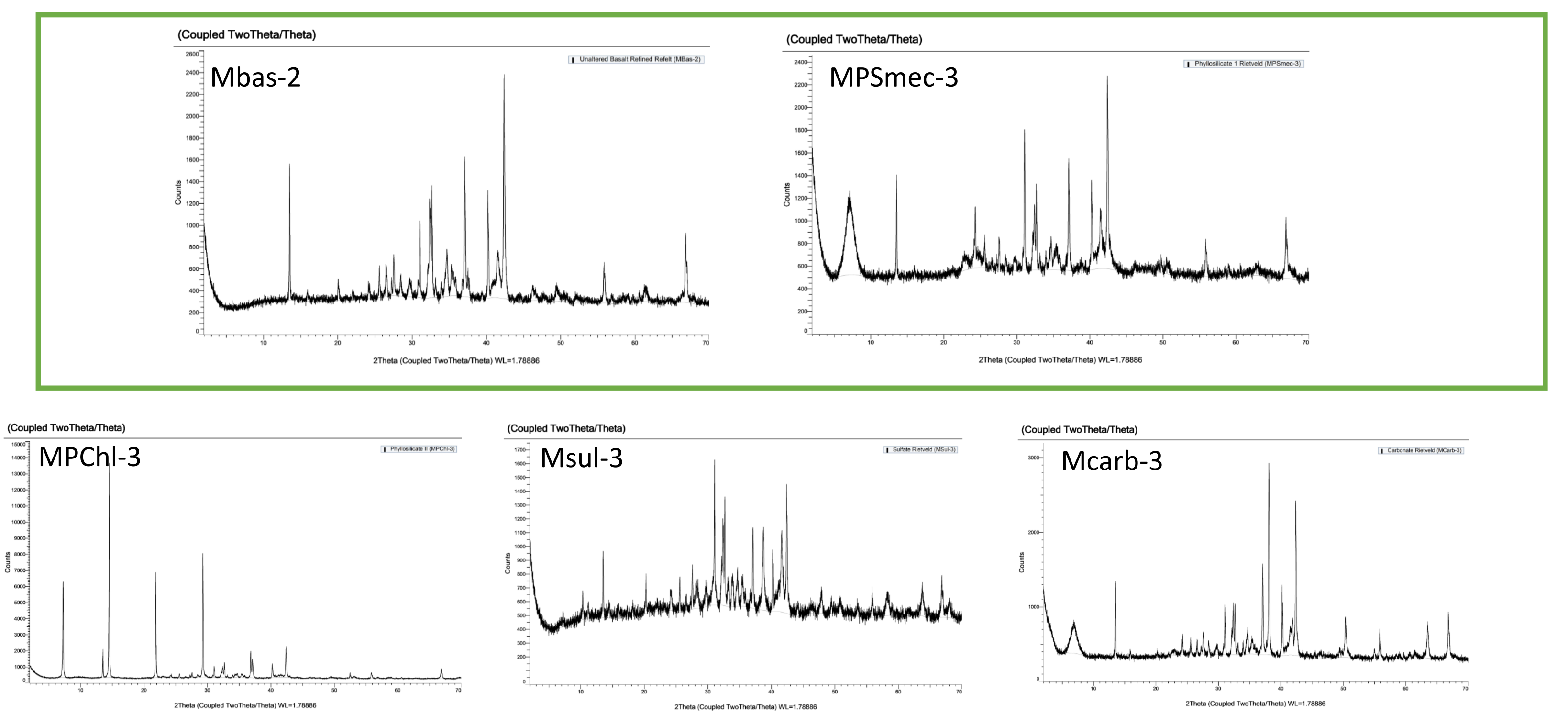
(Coupled TwoTheta/Theta)

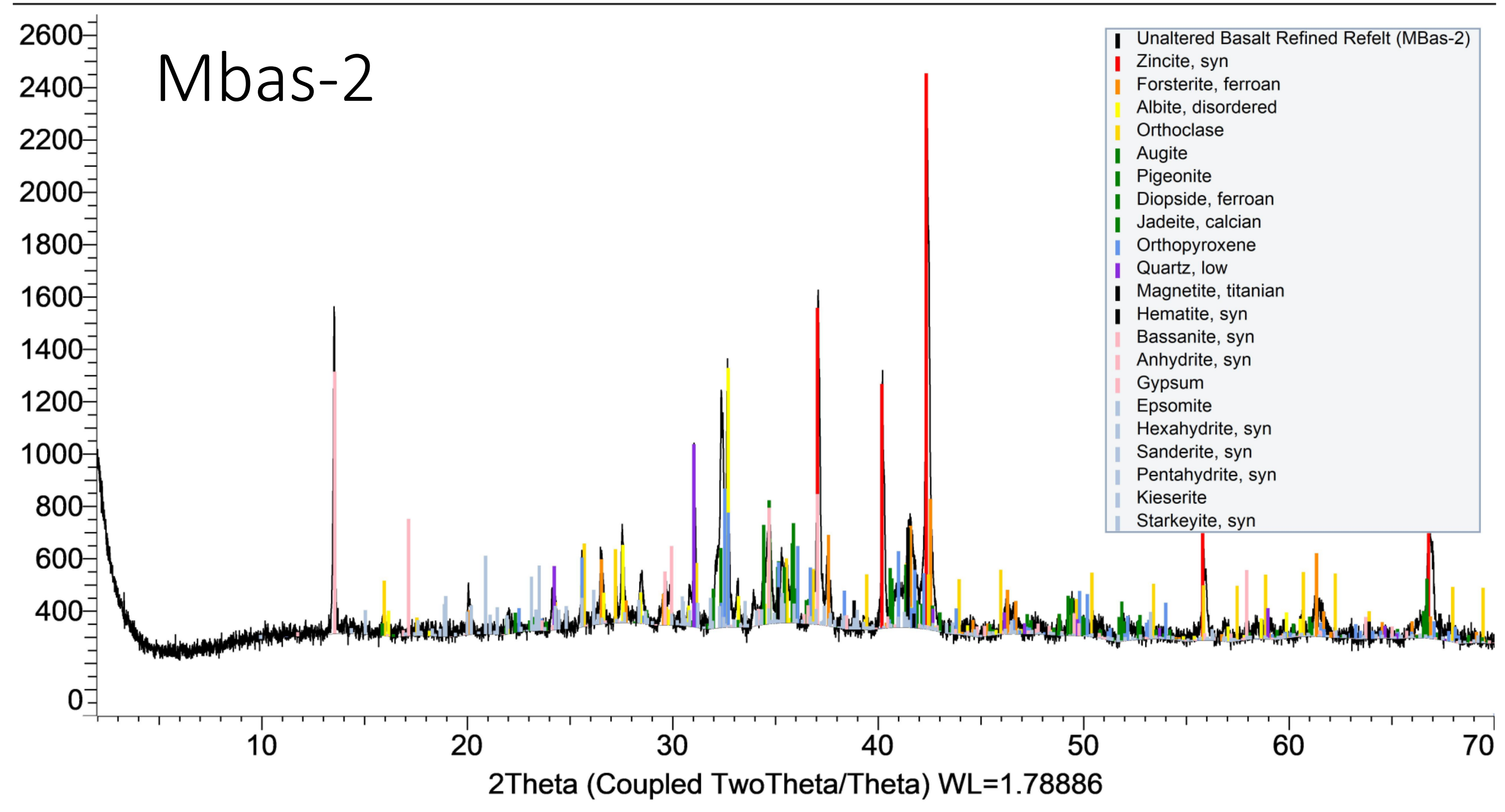




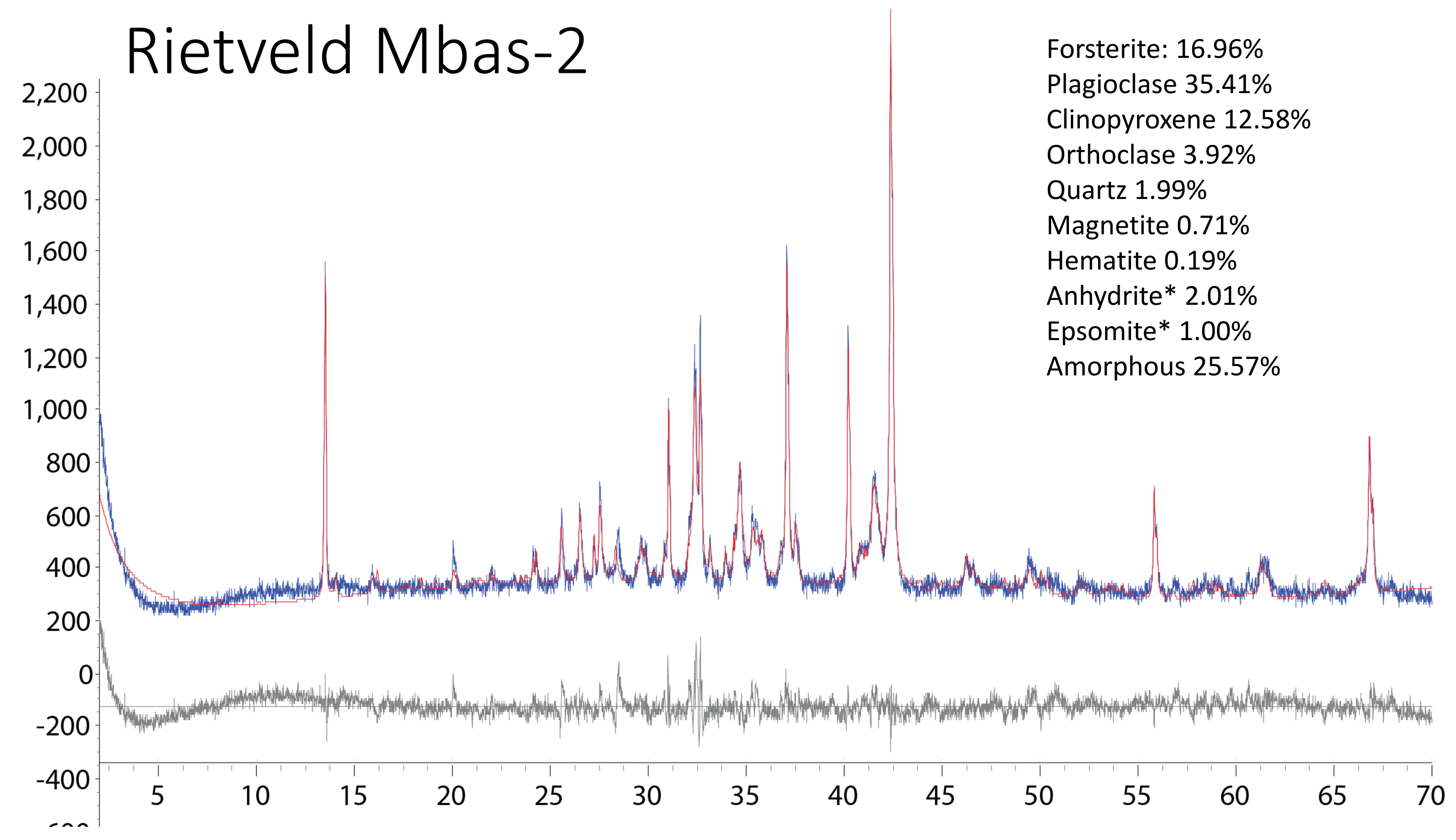




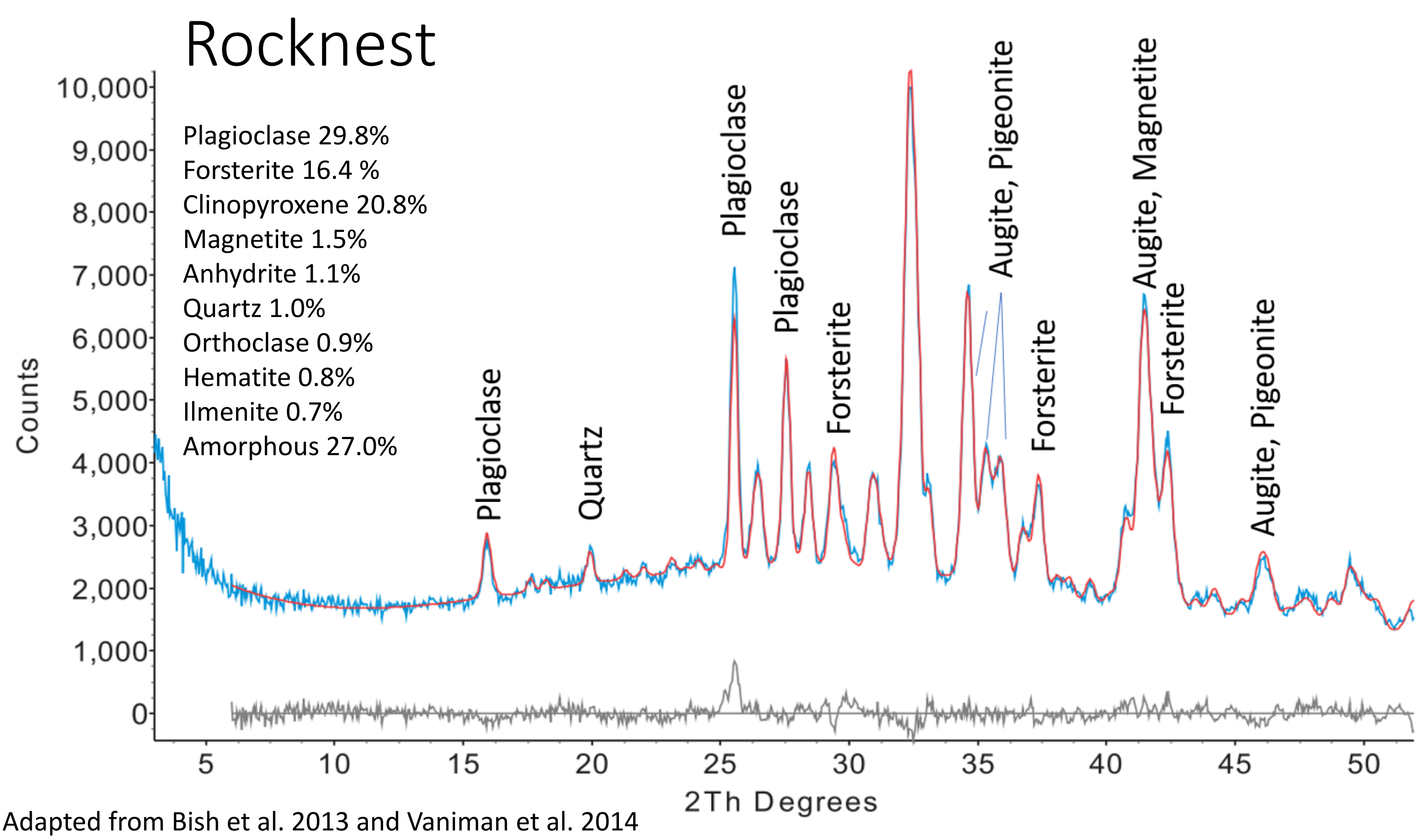




\begin{tabular}{|l|l|l|l|}
\hline & \multicolumn{3}{|c|}{ Regolith 1: Basalt (unaltered)-MBas-2 } \\
\hline Mineral & $\begin{array}{l}\text { Type Locality } \\
\text { Mineralogy (\%) }\end{array}$ & $\begin{array}{l}\text { Simulant } \\
\text { Mineralogy (\%) }\end{array}$ & FOM Score \\
\hline Plagioclase & 29.8 & 35.41 & 0.298 \\
\hline Forsterite & 16.4 & 16.96 & 0.164 \\
\hline Clinopyroxene & 20.8 & 12.58 & 0.1258 \\
\hline Orthopyroxene & & & \\
\hline Magnetite & 1.5 & 0.71 & 0.0071 \\
\hline Gypsum/Anhydrite & 1.1 & 2.01 & 0.011 \\
\hline Bassanite & & & \\
\hline Quartz & 1.0 & 1.99 & 0.01 \\
\hline Orthoclase & 0.9 & 3.92 & 0.009 \\
\hline Hematite & 0.8 & 0.19 & 0.0019 \\
\hline Illmenite & 0.7 & & \\
\hline Goethite & & & 0.2557 \\
\hline Akaganeite & & & \\
\hline Halite & & & \\
\hline Epsomite & & 1.00 & \\
\hline Pyrite & & & \\
\hline Pyrrhotite & & & \\
\hline Smectite & & & \\
\hline Amorphous & 27.0 & & \\
\hline
\end{tabular}
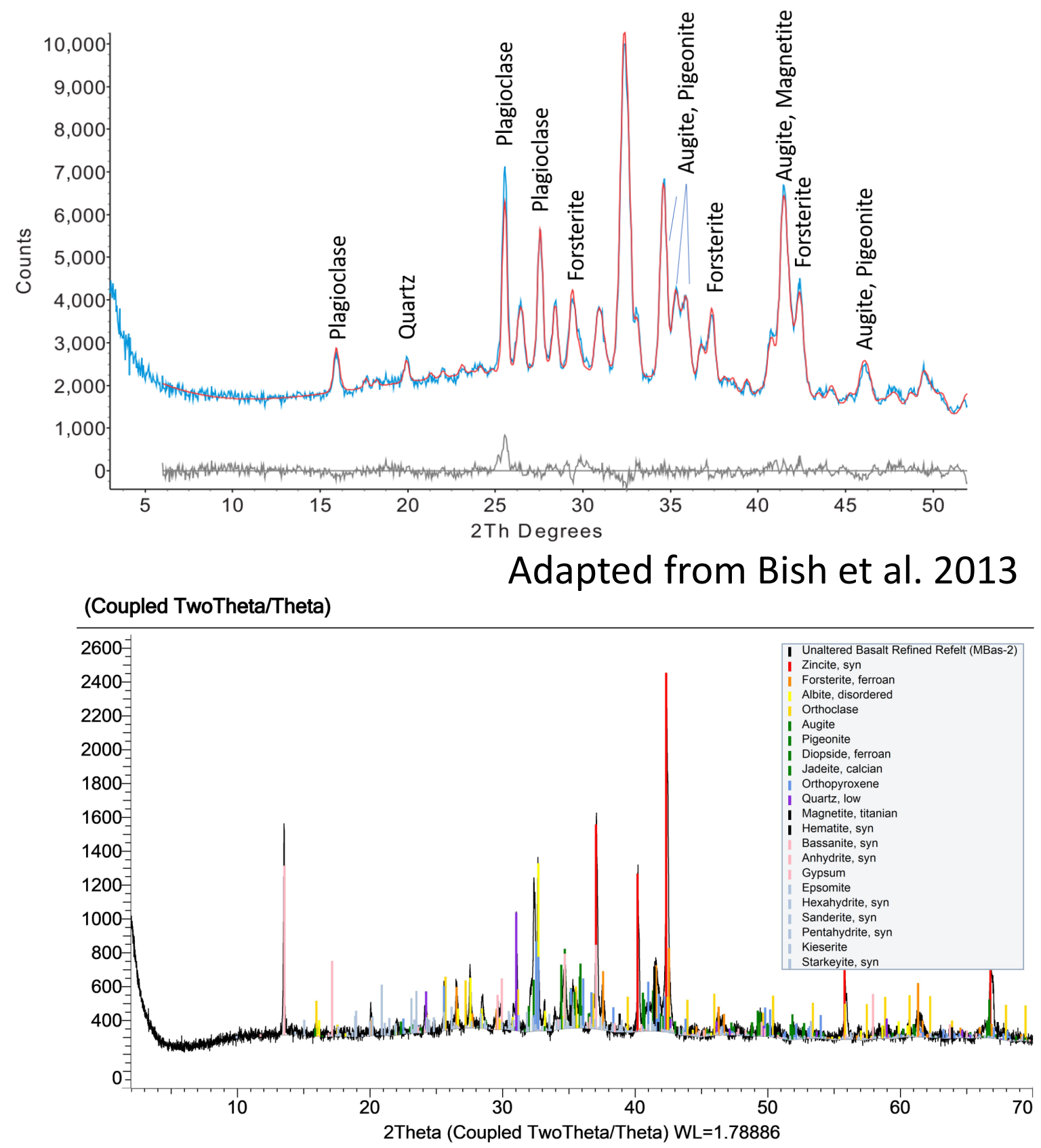


\begin{tabular}{|l|l|l|l|}
\hline & \multicolumn{3}{|c|}{ Regolith l: Basalt (unaltered)-MBas-2 } \\
\hline Mineral & $\begin{array}{l}\text { Type Locality } \\
\text { Mineralogy (\%) }\end{array}$ & $\begin{array}{l}\text { Simulant } \\
\text { Mineralogy (\%) }\end{array}$ & FOM Score \\
\hline Plagioclase & 29.8 & 35.41 & 0.298 \\
\hline Forsterite & 16.4 & 16.96 & 0.164 \\
\hline Clinopyroxene & 20.8 & 12.58 & 0.1258 \\
\hline Orthopyroxene & & & \\
\hline Magnetite & 1.5 & 0.71 & 0.0071 \\
\hline Gypsum/Anhydrite & 1.1 & 2.01 & 0.011 \\
\hline Bassanite & & & \\
\hline Quartz & 1.0 & 1.99 & 0.01 \\
\hline Orthoclase & 0.9 & 3.92 & 0.009 \\
\hline Hematite & 0.8 & 0.19 & 0.0019 \\
\hline Illmenite & 0.7 & & \\
\hline Goethite & & & \\
\hline Akaganeite & & & \\
\hline Halite & & & \\
\hline Epsomite & & 1.00 & \\
\hline Pyrite & & & \\
\hline Pyrrhotite & & & \\
\hline Smectite & & & \\
\hline Amorphous & 27.0 & & \\
\hline & & & \\
\hline
\end{tabular}
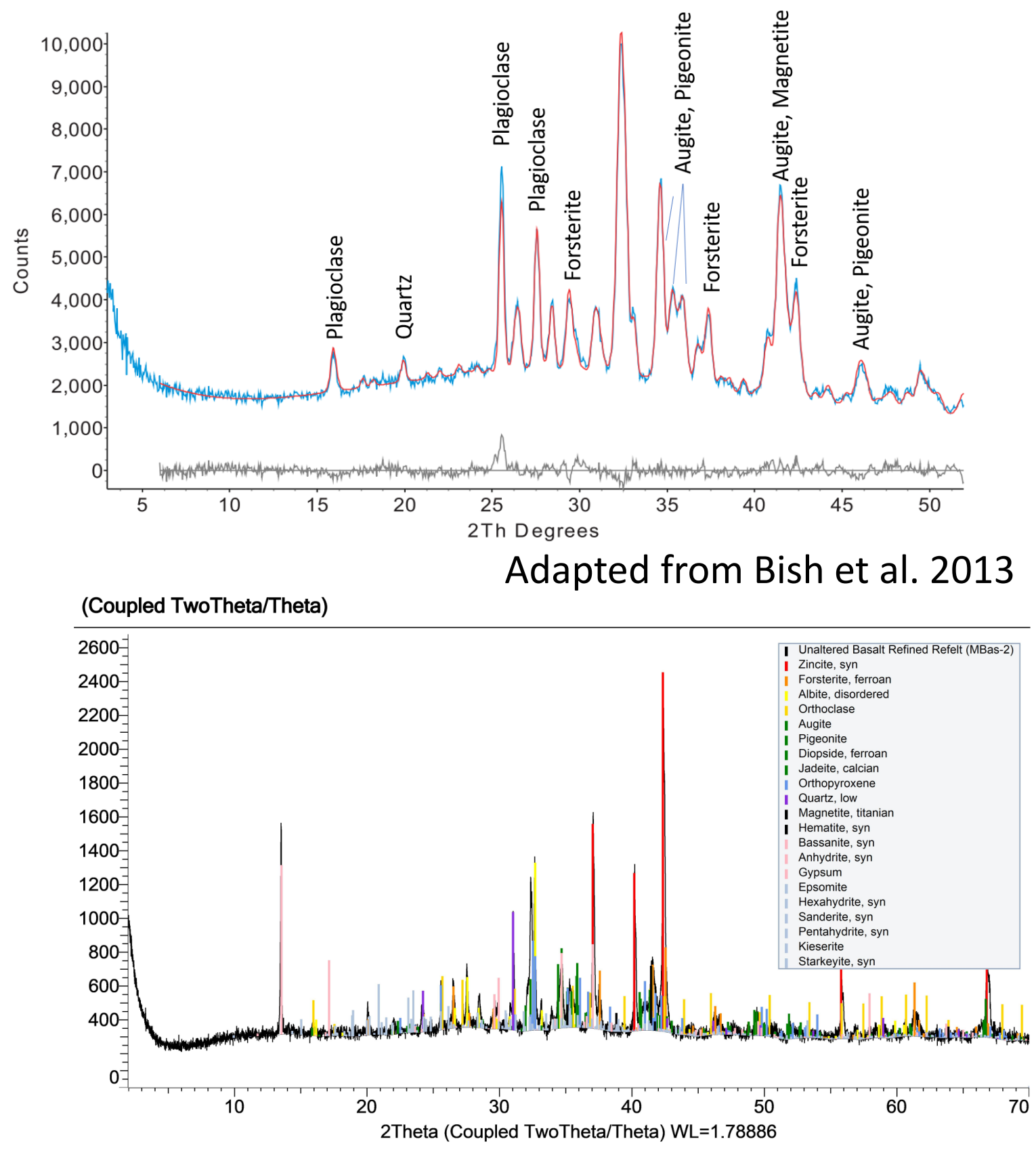


\section{(Coupled TwoTheta/Theta)}

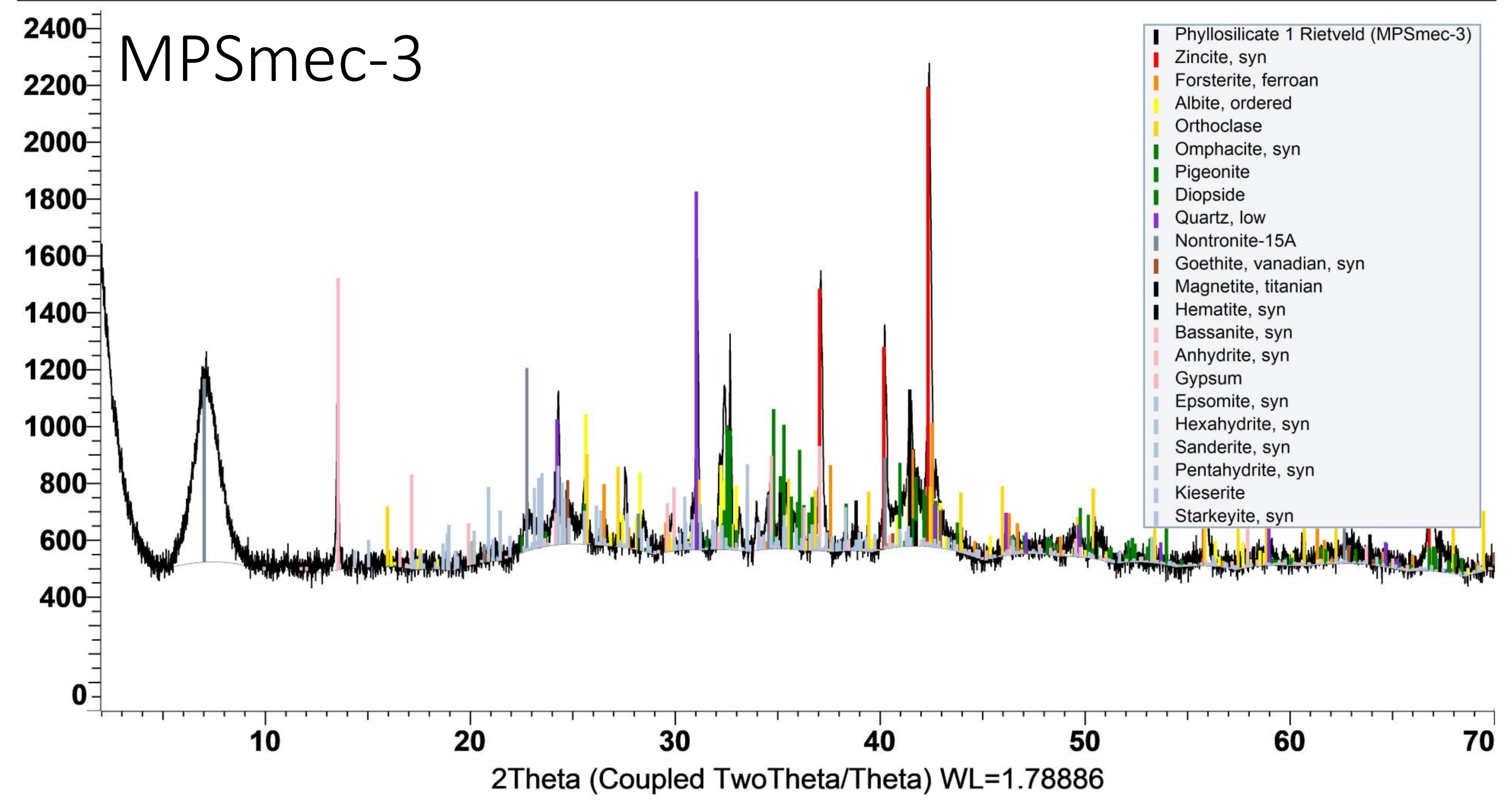




\begin{tabular}{|l|l|l|l|}
\hline Mineral & $\begin{array}{l}\text { Type Locality } \\
\text { Mineralogy (\%) }\end{array}$ & $\begin{array}{l}\text { Simulant } \\
\text { Mineralogy (\%) }\end{array}$ & FOM Score \\
\hline Plagioclase & 22.4 & 19.14 & 0.1914 \\
\hline Forsterite & 2.8 & 1.62 & 0.0162 \\
\hline Clinopyroxene & 9.4 & 9.41 & 0.094 \\
\hline Orthopyroxene & 3.0 & & \\
\hline Magnetite & 3.8 & 3.75 & 0.0375 \\
\hline Gypsum/Anhydrite & 2.6 & 2.04 & 0.0204 \\
\hline Bassanite & 1.1 & & \\
\hline Quartz & 0.4 & 1.62 & 0.004 \\
\hline Orthoclase & 1.2 & 0.97 & 0.0097 \\
\hline Hematite & 0.6 & 1.0 & 0.006 \\
\hline Illmenite & & & \\
\hline Goethite & & 0.42 & \\
\hline Akaganeite & 1.1 & & 0.22 \\
\hline Halite & 0.1 & & \\
\hline Epsomite & & 1.01 & \\
\hline Pyrite & 0.3 & & \\
\hline Pyrrhotite & 1.0 & 28.61 & \\
\hline Smectite & 22 & & \\
\hline Amorphous & 28 & & \\
\hline
\end{tabular}

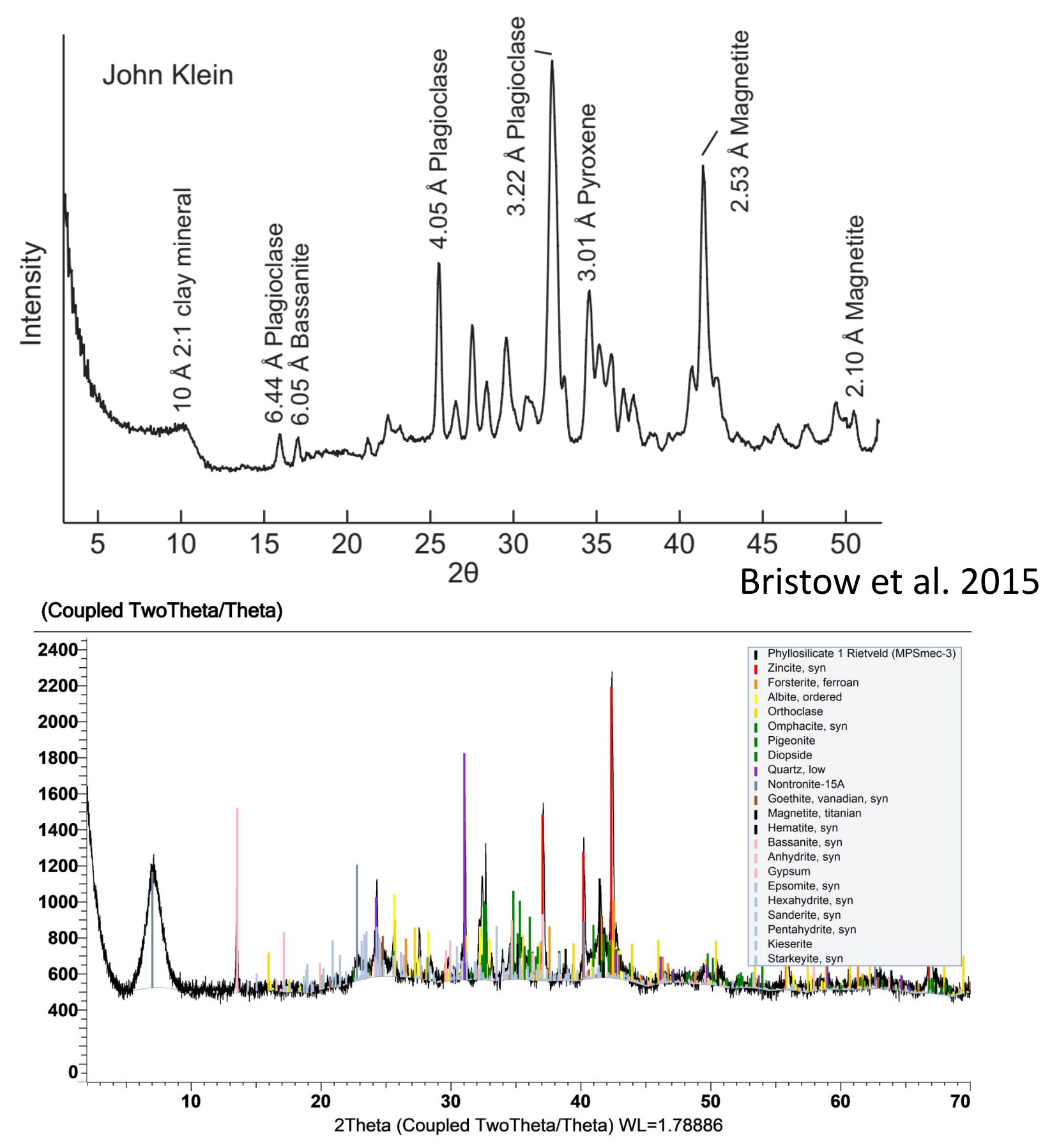




\begin{tabular}{|l|l|l|l|}
\hline Mineral & $\begin{array}{l}\text { Type Locality } \\
\text { Mineralogy (\%) }\end{array}$ & $\begin{array}{l}\text { Simulant } \\
\text { Mineralogy (\%) }\end{array}$ & FOM Score \\
\hline Plagioclase & 22.4 & 19.14 & 0.1914 \\
\hline Forsterite & 2.8 & 1.62 & 0.0162 \\
\hline Clinopyroxene & 9.4 & 9.41 & 0.094 \\
\hline Orthopyroxene & 3.0 & & \\
\hline Magnetite & 3.8 & 3.75 & 0.0375 \\
\hline Gypsum/Anhydrite & 2.6 & 2.04 & 0.0204 \\
\hline Bassanite & 1.1 & & \\
\hline Quartz & 0.4 & 1.62 & 0.004 \\
\hline Orthoclase & 1.2 & 0.97 & 0.0097 \\
\hline Hematite & 0.6 & 1.0 & 0.006 \\
\hline Illmenite & & & \\
\hline Goethite & & 0.42 & \\
\hline Akaganeite & 1.1 & & 0.22 \\
\hline Halite & 0.1 & & \\
\hline Epsomite & & 1.01 & \\
\hline Pyrite & 0.3 & & \\
\hline Pyrrhotite & 1.0 & 28.61 & \\
\hline Smectite & 22 & & \\
\hline Amorphous & 28 & & \\
\hline
\end{tabular}

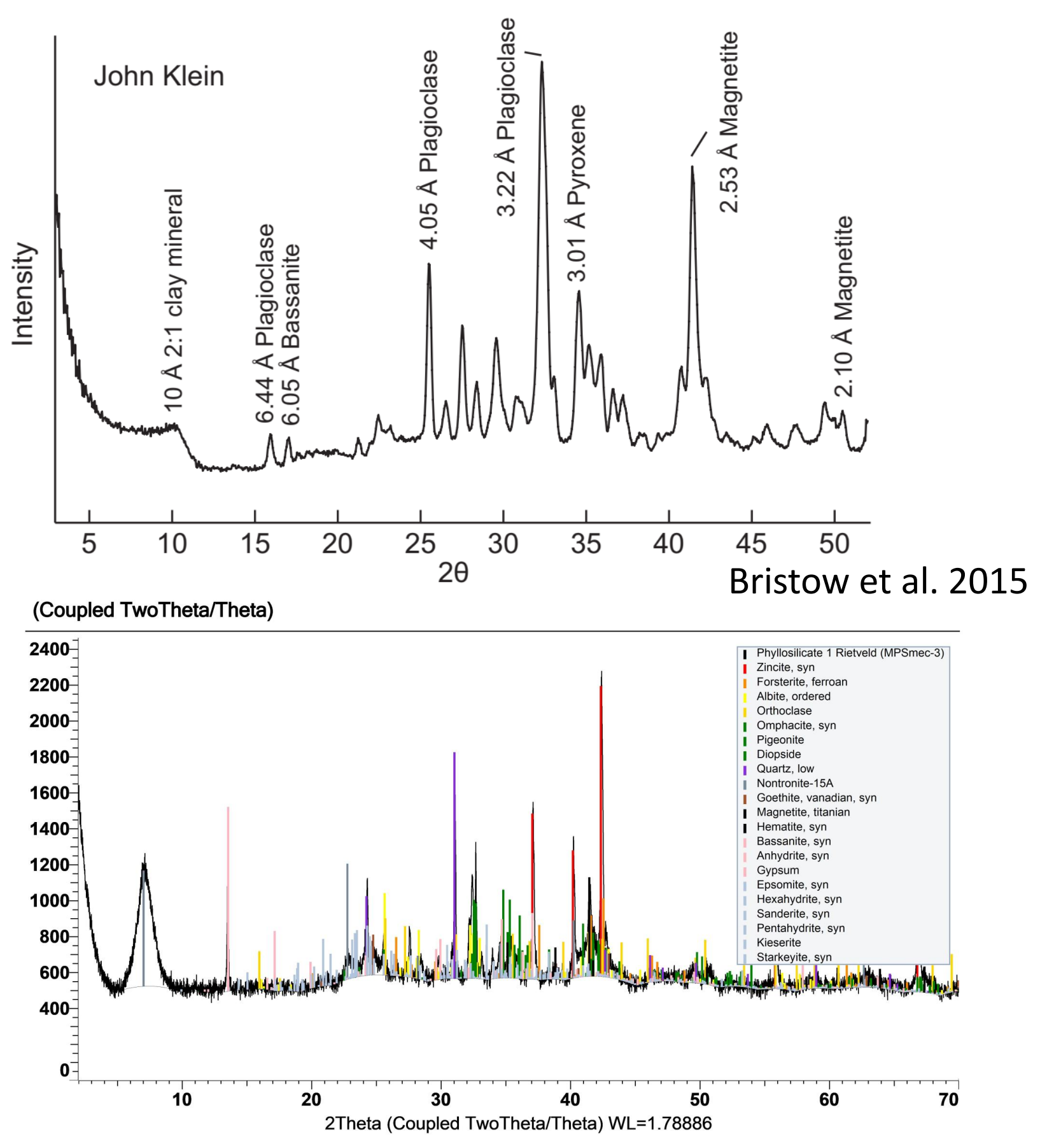




\section{Conclusions}

- Five simulants produced appropriate for the intended application

- Variability of mineralogy to include clays, sulfates and carbonates

- Some important limitations

- These simulants are not made to all characteristics of Mars Soils

- Some characteristics (volatile content, etc..) are assumed to be altered in a 'Martian Greenhouse setting'

- Chlorite and Carbonate regolith not yet studied by rovers, detailed mineralogic and chemical data not available for comparison, spectral data is available 


\section{Acknowledgements}

THE GEOLOGICAL SOCIETY OF AMERICA ${ }^{\circledR}$

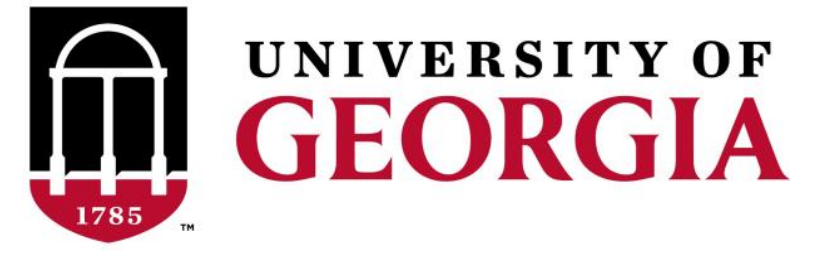

- Research Funding from CMS and UGA Geology

- Travel Funding from UGA graduate school, UGA Geology, and GSA Planetary Division

- Advisor: Paul Schroeder

- Committee: Christian Klimczak, Aaron Thompson, Mussie Habteselassie

- Janice Bishop, David Bish, Doug Archer, Kevin Cannon, Jennifer Thomson, Julie Cox, Kim Seelos, Christina Viviano-Beck, Karl Hibbitts 